\title{
Comparison Between Second Zagreb Eccentricity Index and Eccentric Connectivity Index of Graphs
}

\author{
Huimin $\mathrm{Li}^{1}$, Fang Gao ${ }^{1,2}$ and Kexiang $\mathrm{Xu}^{1 *}$ \\ ${ }^{1}$ College of Science, Nanjing University of Aeronautics \& Astronautics, China \\ ${ }^{2}$ School of Mathematics and Computer Science, Chizhou University, China
}

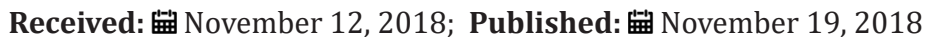

*Corresponding author: Kexiang Xu, College of Science, Nanjing University of Aeronautics \& Astronautics, China

\begin{abstract}
For a graph $\mathrm{G}$, the second Zagreb eccentricity index $\mathrm{E}_{2}(\mathrm{G})$ and eccentric connectivity index $\xi^{c}(G)$ are two eccentricity-based invariants of graph G. In this paper we prove some results on the comparison between $\frac{E_{2}(G)}{m}$ and $\frac{\xi^{c}(G)}{n}$ of connected graphs G of order $\mathrm{n}$ and with $\mathrm{m}$ edges.
\end{abstract}

Keywords: Graph; Eccentricity (of vertex); Second Zagreb eccentricity index; Eccentric connectivity index

\section{Introduction}

Throughout this paper we only consider the finite, undirected, simple and connected graphs. The degree of $v \in V(G)$, denoted by $\operatorname{deg}_{G}(v)$, is the number of vertices in $G$ adjacent to v. For any two vertices $\mathrm{u}$; $\mathrm{v}$ in a graph $\mathrm{G}$, the distance between them, denoted by $d_{G}(u, v)$ is the length of a shortest path connecting them in G. As usual, let $\mathrm{Sn}, \mathrm{Pn}, \mathrm{Cn}, \mathrm{Kn}$ be the star graph, path graph, cycle graph and complete graph, respectively, on $n$ vertices. Other undefined notations and terminology on the graph theory can be found in [1]. For any vertex of graph $G$, the eccentricity $\varepsilon_{G}(v)$ (or $\varepsilon(v)$ for short) is the maximum distance from $v$ to other vertices of $G$, i.e., $\varepsilon_{G}(v)=\max _{\mathrm{u} \neq \mathrm{v}} d_{G}(u, v)$. The eccentricity of a vertex is an important parameter in pure graph theory. The radius of a graph $G$ is denoted by $r(G)$ and defined by $r=r(G)=\{\min \varepsilon(v) \mid v \in V(G)\}$. Also, the diameter of $G$, denoted by $d(G)$, is the maximum distance between vertices of a graph $\mathrm{G}$ and hence $\mathrm{d}=\mathrm{d}(\mathrm{G})=\max \{\varepsilon(v)$ I $\mathrm{v} \in \mathrm{V}(\mathrm{G})\}$. A vertex $\mathrm{v}$ with $\varepsilon_{G}(v)=\mathrm{r}(\mathrm{G})$ is called a central vertex in $G$. A graph $G$ with $d(G)=r(G)$ is called a self-centered graph. A graph which contains only two non-central vertices is called almost self-centered graph [2] (ASC graph for short). Moreover, the eccentricity is also applied in chemical graph theory. There are several eccentricity-based topological indices, including the second Zagreb eccentricity index $\mathrm{E}_{2}(\mathrm{G})$ [3] and eccentric connectivity index $\xi^{c}(G)$ [4], of graphs $G$ where $E_{2}(G)=\sum_{u v \in E(G)} \varepsilon_{G}(u) \varepsilon_{G}(v)$ and $\xi^{c}(G)=\sum_{v \in V(G)} \operatorname{deg}_{G}(v) \varepsilon(v)$.
In particular, we have $\xi^{c}(G)=\sum_{u v \in E(G)}(\varepsilon(u)+\varepsilon(v))$ or any graph $\mathrm{G}$. In this paper we prove some comparison results between $\frac{E_{2}(G)}{m}$ and $\frac{\xi^{c}(G)}{n}$ of connected graphs $\mathrm{G}$ of order $\mathrm{n}$ with m edges.

In this we prove several results on the comparison between $\frac{E_{2}(G)}{m}$ and $\frac{\xi^{c}(G)}{n}$ of graphs G. Firstly we present two useful lemmas.

Lemma 2.1. ([5]) Let $G$ be a connected graph of order $n$ with maximum degree $\Delta$. If $\Delta=n-1$ then $E_{2}(G)=\xi^{c}(G)$ .Otherwise, $E_{2}(G) \geq \xi^{c}(G)$ with equality holds if and only if $\mathrm{G}$ is a 2-SC graph.

Lemma 2.2. ([6]) If $u$ and $v$ are two adjacent vertices of a connected graph $\mathrm{G}$, then $|\varepsilon(u)-\varepsilon(v) \leq 1|$.

Denote by $\zeta_{n}(m ; d)$ the set of connected graphs of order $\mathrm{n}$ with $\mathrm{m}$ edges and diameter $\mathrm{d}$.

Theorem 2.3. Let $G \in \zeta_{n}(m, d)$ with $\mathrm{n}>5$ and $d \leq 2$. Then $\frac{E_{2}(G)}{m}<\frac{\xi^{c}(G)}{n}$. Proof. If d $=1, G \in \zeta_{n}(m, d)$ contains a single graph Kn with ${ }^{n}=\left(\begin{array}{l}n \\ 2\end{array}\right)$ and $E_{2}\left(K_{n}\right)=\left(\begin{array}{l}n \\ 2\end{array}\right)<n(n-1)=\xi^{c}\left(K_{n}\right)$. Then our result follows. Next it suffices to consider the case when $d=2$. If $G$ has maximum degree $\Delta=n-1$ by Lemma 2.1 , we have $E_{2}(G)<\xi^{c}(G)$ for any graph $G \in \zeta_{n}(m, d)$.Moreover, we have $m \geq n-1$ If $\mathrm{m}=$ n-1, then $G \cong S_{n}$ with $\frac{E_{2}(G)}{m}=2<\frac{\xi^{c}(G)}{n}=\frac{3(n-1)}{n}$ for any $\mathrm{n} \geq 5$. 
Moreover, $\frac{E_{2}(G)}{m}<\frac{\xi^{c}(G)}{n}$ holds clearly form $\geq$ n. If $\Delta \leq n-2$ then $\mathrm{G}$ is a 2-SC graph. By Lemma 2.2, G is never a tree. Therefore $\mathrm{m} \geq \mathrm{n}$ with equality holding if and only if $G \cong C_{4}$ or $G \cong C_{5}$ . Consider that $\mathrm{n}>5, \mathrm{~m}>\mathrm{n}$ holds immediately. It follows that $\frac{E_{2}(G)}{m}=4<\frac{4 m}{n}=\frac{2 \sum_{u v \in V(G)} \operatorname{deg}_{G}(v)}{n}=\frac{\xi^{c}(G)}{n}$. This completes the proof of the theorem.

In the following we consider the graphs $G \in \zeta_{n}(m, d)$ with diameter $\mathrm{d} \geq 3$.

Theorem 2.4. Let $G \in \zeta_{n}(m, d)$. with $\mathrm{d} \geq 3, \mathrm{n}>5$ be a tree or a unicyclic graph. Then $\frac{E_{2}(G)}{m}>\frac{\xi^{c}(G)}{n}$

Proof. If $\mathrm{d} \geq 3$, then $\Delta(G) \leq n-2$. From Lemma 2.1. we have $E_{2}(G)>\xi^{c}(G)$. Note that $\mathrm{m} \leq \mathrm{n}$ for any tree or unicyclic graph G. Thus, it follows $\frac{E_{2}(G)}{m} \geq \frac{E_{2}(G)}{n}>\frac{\xi^{c}(G)}{n}$. finishing the proof of the theorem. Next, we consider the case $\mathrm{m}>\mathrm{n}$. In the following theorem we give a sufficient condition for the graph $\mathrm{G}$ of order $\mathrm{n}$ with $\frac{E_{2}(G)}{m} \geq \frac{\xi^{c}(G)}{n}$.

Theorem 2.5. Let $G \in \zeta_{n}(m, d)$ with $\mathrm{d} \geq 3, \mathrm{~m}=\mathrm{n}+\mathrm{t}$ and $1 \leq t \leq \frac{n}{2}$ . If $r(G) \geq 3$, then $\frac{E_{2}(G)}{m} \geq \frac{\xi^{c}(G)}{n}$, with equality holding if and only if $G$ is a self-centered graph with $r(G)=3$.

Proof. Making a difference, we have

$$
\frac{E_{2}(G)}{m}-\frac{\xi^{c}(G)}{n}=\frac{n E_{2}(G)-(n+t) \xi^{c}(G)}{n(n+t)} .
$$

Set $\Delta_{1}=n E_{2}(G)-(n+t) \xi^{c}(G)$. From Lemma 2.2, we have

$$
\begin{aligned}
& \Delta_{1}=\sum_{u v \in E(G)}[n \varepsilon(u) \varepsilon(v)-(n+t)(\varepsilon(u)+\varepsilon(v))] \\
& \Delta_{1}=\sum_{u v \in E(G), \varepsilon(v)=\varepsilon(u)}\left[n \varepsilon^{2}(u)-2 \varepsilon(u)(n+t)\right]+ \\
& \sum_{u v \in E(G), \varepsilon(v)=\varepsilon(u)+1}[n \varepsilon(u)(\varepsilon(u)+1)-(n+t)(2 \varepsilon(u)+1)]
\end{aligned}
$$

Since $r(G) \geq 3$ and $1 \leq t \leq \frac{n}{2}$, we have

$n \varepsilon^{2}(u)-2 \varepsilon(u)(n+t) \geq 9 n-6(n+t)=3(n-2 t) \geq 0, n \varepsilon(u)(\varepsilon(u)+1)-(n+t)(2 \varepsilon(u)+1)$ $=\varepsilon(u)[n \varepsilon(u)-n-2 t]-n-t \geq 3(2 n-2 t)-n-t=5 n-7 t>0$

Therefore, $\Delta_{1} \geq 0$ with equality holding if and only if $\varepsilon(u)=3$ for each vertex $u \in V(G)$ that is, $\mathrm{G}$ is a self-centered graph with radius 3 . This completes the proof of the theorem.

For, $G \in \zeta_{n}(m, d)$ with $\mathrm{d} \geq 3, \mathrm{r}=2$ and $1 \leq t \leq \frac{n}{2}$ considering that $r(G) \leq d(G) \leq 2 r(G)$ we have

$\mathrm{d}(\mathrm{G})=3$ or $\mathrm{d}(\mathrm{G})=4$. In this case, the value of $\Delta_{1}$ may be negative, zero or positive. Let $\varepsilon_{1}=\{u v: u v \in E(G), \varepsilon(u)=\varepsilon(v)=2\}, \varepsilon_{2}=\{u v: u v \in E(G), \varepsilon(u)=2, \varepsilon(v)=3\}$, $\varepsilon_{3}=\{u v: u v \in E(G), \varepsilon(u)=\varepsilon(v)=3\}, \varepsilon_{4}=\{u v: u v \in E(G), \varepsilon(u)=3, \varepsilon(v)=4\}$, $\varepsilon_{5}=\{u v: u v \in E(G), \varepsilon(u)=\varepsilon(v)=4\}$.

Denote by $\mathrm{m}_{\mathrm{i}}$ the cardinality of $\varepsilon_{i}$ and $i \in\{1,2,3,4,5\}$. Then

$$
\begin{aligned}
& \Delta_{1}=\sum_{u v E(G)}[n \varepsilon(u) \varepsilon(v)-(n+t)(\varepsilon(u)+\varepsilon(v))] \\
& \Delta_{1}=-4 t m_{1}+(n-5 t) m_{2}+(3 n-6 t) m_{3}+(5 n-7 t) m_{4}+(8 n-8 t) m_{5}
\end{aligned}
$$

In the following result we present some comparison results for ASC graphs.

Theorem 2.6. Let $G \in \zeta_{n}(m, d)$ with $\mathrm{d}=3, \mathrm{r}=2, \mathrm{~m}=\mathrm{n}+\mathrm{t}, \mathrm{t} \geq 1$ where $n<\frac{5 t+2 \sqrt{41 t^{2}+12 t+4}}{2}$. If G is an ASC graph, then $\frac{E_{2}(G)}{m}<\frac{\xi^{c}(G)}{n}$

Proof. If $\mathrm{G}$ is an ASC graph with $\mathrm{d}=3, \mathrm{r}=2$, from the structure of ASC graph, we have $m_{2} \leq n-2, m_{3}=0$, that is, $m_{1} \geq t+2$. If $n \leq 5 t$, clearly, we have $\Delta_{1} \leq 0$. For $\mathrm{n}>5 \mathrm{t}$, we have

$$
\begin{aligned}
& \Delta_{1} \leq-4 t(t+2)+(n-5 t)(n-2) \\
& =n^{2}-(5 t+2) n-2 t(2 t-1) \\
& <0
\end{aligned}
$$

holds if and only if $\frac{5 t+2-\sqrt{41 t^{2}+12 t+4}}{2}<n<\frac{5 t+2+\sqrt{41 t^{2}+12 t+4}}{2}$ Note that

$\frac{5 t+2-\sqrt{41 t^{2}+12 t+4}}{2}<0$ Thus $\Delta_{1}<0 \quad$ is equivalent that

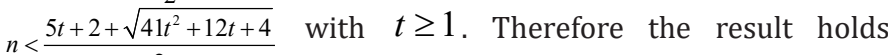
immediately.

It is much interesting to search for more generalized graphs $G$ with different comparison results between $\frac{E_{2}(G)}{m}$ and $\frac{\xi^{c}(G)}{n}$ which can be a topic for further research in the future.

\section{References}

1. JA Bondy, USR Murty (1976) Graph Theory with Applications. Macmillan Press, New York, 1976.

2. S Klavzar, KP Narayankar, HB Walikar (2011) Almost self-centered graphs. Acta Math Sin (Engl Ser) 27(12): 2343-2350.

3. D Vukicevic, A Graovac (2010) Note on the comparison of the first and second normalized Zagreb eccentricity indices. Acta Chim Slov 57(3): 524-528.

4. V Sharma, R Goswami, AK Madan (1997) Eccentric connectivity index: A novel highly discriminating topolog-ical descriptor for structureproperty and structure-activity studies. J Chem Inf Comput Sci 37(2): 273-282.

5. KC Das (2016) Comparison between Zagreb eccentricity indices and the eccentric connectivity index, the second geometric-arithmetic index and the graovac-ghorbani index. Croat Chem Acta 89(4): 505-510.

6. M Behzad, JE Simpson (1976) Eccentric sequences and eccentric sets in graphs. Discrete Math 16(3) 187-193. 
(c) (i) This work is licensed under Creative

To Submit Your Article Click Here: Submit Article

DOI: $10.32474 /$ CTCSA.2018.01.000105

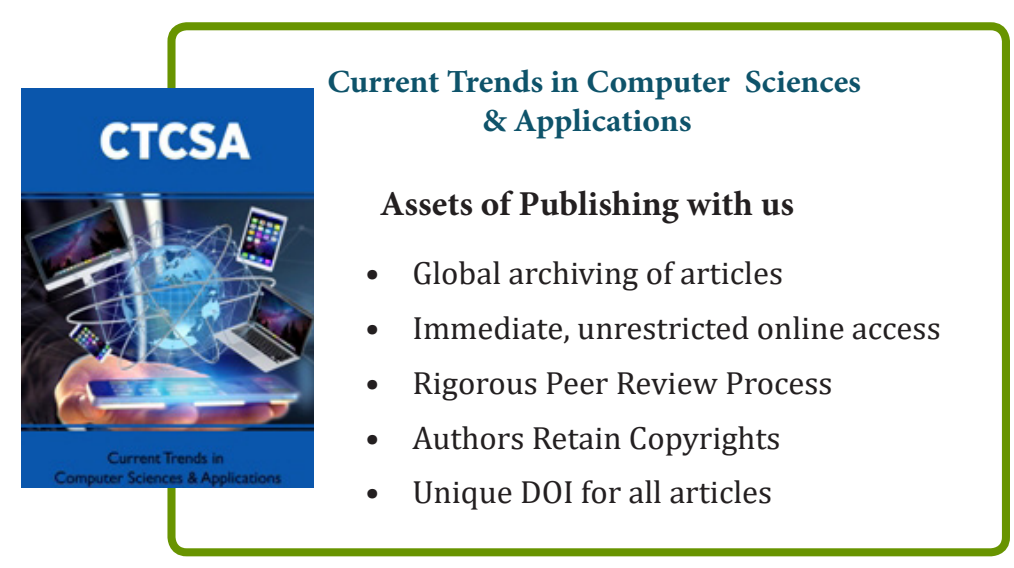

